\title{
Microwave Analysis for Intrinsic and Extrinsic Characteristics of AlGaN/GaN pHEMT
}

\author{
Ramnish $^{1}$, Sandeep K Arya ${ }^{1}$ and Anil Ahlawat ${ }^{2}$ \\ ${ }^{1}$ Department of Electronics and Communication Engineering, \\ Guru Jambheshwar University of Science and Technology, Hisar, Haryana, India \\ ${ }^{2}$ Department of Computer Science Engineering, \\ Krishna Institute of Engineering and Technology, Ghaziabad, U.P., India \\ contactram1@rediffmail.com
}

\begin{abstract}
In this paper, a simple non-linear analytical charge control model for the DC and microwave characteristics of AlGaN/GaN MODFET is presented. The effect of parasitic resistances $R_{s}$ and $R_{d}$ is also incorporated. The model has also been extended to obtain the expressions for transconductance, drain conductance and cut-off frequency of the device. The model predicts a high transconductance of $502.6 \mathrm{~mA} / \mathrm{mm}$ at 1 Vof gate bias and a maximum cut-off frequency of $22.5 \mathrm{GHz}$ for a 50nm device gate length, which is important in realizing the device for microwave applications. The extrinsic and intrinsic characteristics show close agreement with the published results proving the validity of the model.
\end{abstract}

Key-words: AlGaN/GaN MODFET, parasitic resistances, extrinsic and intrinsic characteristics

\section{Introduction}

High Electron Mobility Transistor (HEMT) or Modulation Doped field Effect Transistor (MODFET) is the fastest transistor currently available and a suitable candidate for microwave and millimeter wave applications [1-2]. It is a hetero-structure device and the unique properties of HEMT are the presence of a two dimensional electron gas (2-DEG) at its interface [3]. Because of its high power handling capabilities, GaN based pHEMTs have attracted increasing interests [4-5]. Owing to its large band-gap, $\mathrm{GaN}$ is particularly suitable for high frequency, high power and high temperature applications [6-7]. In addition, $\mathrm{AlGaN} / \mathrm{GaN}$ material is suitable for applications in the transmission and distribution of electric power [8-9]. The AlGaN/GaN pHEMT is an attractive option for various radar, communication and satellite applications in the high frequency range [10-11]. AlGaN/GaN pHEMTs have breakdown voltages in excess of $100 \mathrm{~V}$, which eliminates the need for protection circuitry, such as in a front-end receiver, making an $\mathrm{AlGaN} / \mathrm{GaN}$ based design less complex with lower noise [12]. AlGaN/GaN has also lower electron mobility and high electron velocity making it useful at high frequencies. Since, these values of electron mobility and electron velocity translate into a good unity current gain $\left(\mathrm{f}_{\mathrm{T}}\right)$ and maximum frequency of oscillation $\left(f_{\max }\right)$, so, it also performs well for noise [13]. The short channel effects and parasitic resistances deteriorate the microwave performance of the device. So, it is significant to figure out the extrinsic and intrinsic factors behind the electrical degradation of 
$\mathrm{AlGaN} / \mathrm{GaN}$ pHEMTs. In the present work, these factors have been improved to use this device over a wide range of operation. [14-15].

In the present work, a non-linear DC model for AlGaN/GaN MODFET is developed, based on the $\mathrm{AlGaAs} / \mathrm{GaAs}$ system proposed by $\mathrm{N}$. Dasgupta et al. [16] which is used to formulate the I-V characteristics from sub-threshold to high conduction region. The effects of source and drain resistances have also been included in order to find the extrinsic characteristics. Both intrinsic and extrinsic small-signal parameters such as transconductance, drain/output conductance and cut-off frequencies have also been evaluated. The present model is valid in the linear region and can be extended in the saturation region also.

\section{Model Formulation}

In this section, an analytical model of a $\mathrm{AlGaN} / \mathrm{GaN}$ MODFET is presented. The basic structure of the $\mathrm{AlGaN} / \mathrm{GaN}$ MODFET for the present work is shown in Figure1.

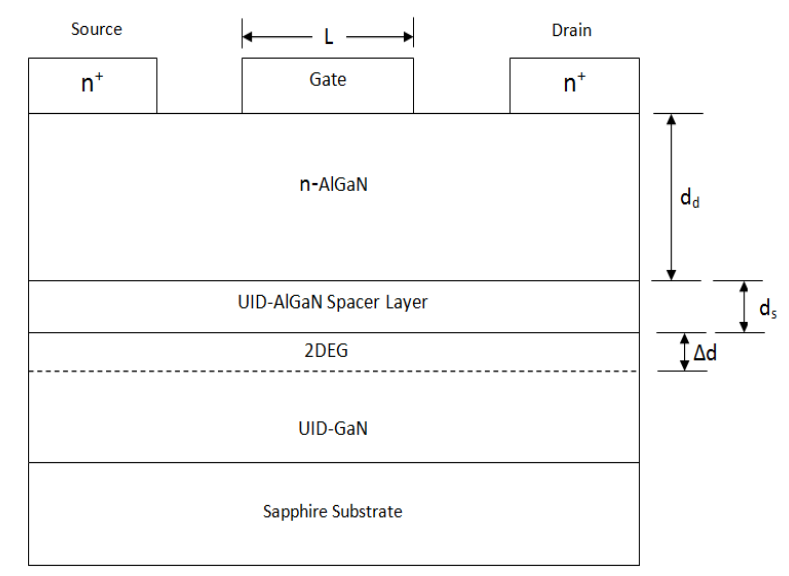

Figure 1. Cross-sectional view of 2-DEG MODFET

The threshold voltage $V_{t h}$ of the doped structure can be expressed as:

$V_{t h}=\Phi_{m}-\Delta E_{c}-\frac{q N_{d} d_{d}^{2}}{2 . \varepsilon}\left(1+\frac{2 . d_{s}}{d_{d}}\right)$

where $\Phi_{m}$ is the Schottky barrier height, $\Delta E_{c}$ is the conduction band discontinuity at heterojunction, $N_{d}$ doping density of the AlGaN layer, $d=d_{d}+d_{s}+\Delta_{d}$ is the separation between the gate and the channel, $d_{d}$ is the doped AlGaN layer thickness, $d_{s}$ is the undoped AlGaN spacer layer thickness, $\Delta_{d}$ is the effective width of the 2DEG. In the foregoing section, an attempt is made to obtain the various parameters of the proposed model such as transconductance, drain conductance etc. analytically by taking the parasitic resistances into account.

\subsection{Intrinsic Characteristics}

The drain current in the channel for AlGaN/GaN MODFET can be written [17] as:

$$
I_{d}=q Z n_{s}(x) v(x)
$$


Where $\mathrm{q}$ is the electron charge, $\mathrm{Z}$ is the gate width and $v(x)$ is the electron charge carrier velocity, $n_{s}(x)$ is the 2-DEG sheet charge carrier concentration in an AlGaN/GaN MODFET and is given as:

$n_{s}(x)=\frac{\varepsilon(m)}{q d}\left\{V_{g s}-V_{t h}-V_{c}(x)\right\}$

Where $\varepsilon(m)$ is the dielectric constant, $\mathrm{m}$ is the $\mathrm{Al}$ mole fraction, $V_{g s}$ is the gate-source voltage, $V_{c}(x)$ is channel potential at any point $x$ due to drain voltage.

For simplicity, the following velocity-field relation is assumed to be valid for carrier transport in the channel:

$$
\begin{gathered}
v(x)=\frac{\mu_{0} \frac{d v(x)}{d x}}{1+\frac{1}{E_{c}} \frac{d v(x)}{d x}}{\text { for } E_{x}<E_{c}}^{=V_{\text {sat }} \quad \text { for } E_{x}>E_{c}}
\end{gathered}
$$

Where $E_{x}=\frac{d v(x)}{d x}$ is the electric field at any point $x$ in the channel, $V_{c}(x)$ is the channel potential at point $x$ due to drain voltage, $\mu_{o}$ is the low field mobility, and $E_{c}$ is the critical field given by $E_{c}=V_{s a t} / \mu_{o}$ and $V_{s a t}$ is the saturation velocity of the electrons. .

\subsubsection{Linear Region}

The electron velocity is less than the saturation velocity $V_{\text {sat }}$ for lower drain voltages. By substituting eq. (2) in eq.(1) for $E_{x}<E_{c}$ and using eq.(3), the drain current in the linear region can be obtained as:

$$
I_{d s}=\frac{q Z \mu_{0} \frac{d v(x)}{d x}}{1+\frac{1}{E_{c}} \frac{d v(x)}{d x}}\left[\frac{\varepsilon(m)}{q d}\left\{V_{g s}-V_{t h}-V_{c}(x)\right\}\right]
$$

After solving eq. (5) using boundary conditions $V(x)=0$ for $x=0$ and $V(x)=L$ for $x=L$, the current flowing through the $2 \mathrm{DEG}$ is found to be:

$$
I_{d s}=\frac{\varepsilon Z \mu_{0}}{d\left(L+\frac{V_{d s}}{E_{c}}\right)}\left[\left(V_{g s}-V_{t h}\right) V_{d s}-\frac{V_{d s}^{2}}{2}\right]
$$

Where $\mathrm{L}$ is the gate length of the device.

2.1.2. Saturation Region: The channel voltage increases from source to drain, the electric field is maximum close to the drain, and the velocity saturation occurs first at the drain side of 
the gate region. At the drain end of the channel, the velocity of the electrons saturates to $V_{\text {sat }}$. Thus from eq. (4) for $E_{x}>E_{c}$, the drain current can be given as:

$$
I_{d s a t}=q Z n_{s}(x) v_{s a t}
$$

Using eq. (4) and eq. (7), with $V_{c}(x)=V_{d s a t}, I_{d s a t}$ is given as:

$$
I_{d s a t}=\frac{Z \varepsilon v_{s a t}}{d}\left(V_{g s}-V_{t h}-V_{d s a t}\right)
$$

The saturation drain voltage can be calculated analytically by equating eq. (8) and eq. (6) with $V_{d}=V_{d s a t}$ as:

$$
\begin{aligned}
& A_{1} V_{d s a t}^{2}+A_{2} V_{d s a t}^{2}+A_{3}=0 \text { or } \\
& V_{d s a t}=\frac{-A_{2}+\sqrt{A_{2}^{2}-4 A_{1} A_{3}}}{2 A_{1}}
\end{aligned}
$$

Where

$$
A_{1}=\frac{\mu E_{c}-2 v_{s a t}}{2 E_{c}}, A_{2}=\left(V_{g s}-V_{t h}\right)\left(\frac{V_{s a t}-\mu E_{c}}{E_{c}}\right)-L V_{s a t} \text { and } A_{3}=\left(V_{g s}-V_{t h}\right) L v_{s a t}
$$

2.1.3. Transconductance: It is a measure of device gain and cut-off frequency. Mathematically, it is given as:

$$
\mathrm{g}_{\mathrm{m}}=\left.\frac{\partial I_{d s}}{\partial V_{g s}}\right|_{V_{d s}}
$$

Transconductance in linear region can be obtained by differentiating eq. (6) w.r.t. $V_{g s}$.

$$
g_{m}=\frac{Z \mu \varepsilon V_{d s}}{d\left(L+\frac{V_{d s}}{E_{c}}\right)}
$$

2.1.4. Drain Conductance: It is also a measure of the device gain. Mathematically, it is given as:

$$
\mathrm{g}_{\mathrm{d}}=\left.\frac{\partial I_{d s}}{\partial V_{d s}}\right|_{V_{g s}}
$$

Drain conductance in linear region can be obtained by differentiating eq. (6) w.r.t. $V_{d}$

$$
g_{d}=\frac{Z \mu_{0} \varepsilon}{d\left(L+\frac{V_{d s}}{E_{c}}\right)}\left(V_{g s}-V_{t h}-V_{d s}\right)+\left\{\left(V_{g s}-V_{t h}\right) V_{d s}-\frac{V_{d s}^{2}}{2}\right\}\left\{-\frac{1}{E_{c}}\left(\frac{Z \mu_{0} \varepsilon}{d\left(L+\frac{V_{d s}}{E_{c}}\right)^{2}}\right)\right\}
$$




\subsection{Extrinsic Characteristics}

As the gate length approaches the submicron region, parasitic resistances become comparable with channel resistances and can't be neglected. Incorporating the parasitic resistances $R_{s}$ and $R_{d}$ into account, the gate and drain voltages are thus modified as follows:

$V_{d s} \rightarrow V_{d s}-I_{d s}\left(R_{s}+R_{d}\right)$

$V_{g s} \rightarrow V_{g s}-I_{d s} R_{s}$

2.2.1. Linear region: Considering the above conditions, the drain current in the linear region can be obtained from eq. (8) as:

$$
\begin{aligned}
& B_{1} I_{d s}^{2}+B_{2} I_{d s}+B_{3}=0 \\
& I_{d s}=\frac{-B_{2}+\sqrt{B_{2}^{2}-4 B_{1} B_{3}}}{2 B_{1}}
\end{aligned}
$$

Where

$$
\begin{aligned}
& B_{1}=\left(R_{s}+R_{d}\right)\left\{\frac{d}{E_{c}}+\frac{\varepsilon \mu Z}{2}\left(R_{s}-R_{d}\right)\right\} \\
& B_{2}=-d L-\frac{d}{E_{c}} V_{d s}-\varepsilon \mu Z\left(R_{s}+R_{d}\right)\left[V_{g s}-V_{t h}-V_{d}\right]-\varepsilon \mu Z R_{s} V_{d}
\end{aligned}
$$

And $B_{3}=\varepsilon \mu Z\left(V_{g s} V_{d s}-V_{t h} V_{d s}-\frac{V_{d s}^{2}}{2}\right)$

2.2.2. Saturation region: The drain current in saturation region can be obtained analytically from eq. (8) with $V_{d}=V_{d s a t}$,

$$
I_{d s a t}=\frac{Z \varepsilon v_{s a t}}{d}\left(V_{g s}-V_{t h}-V_{d s a t}+I_{d s a t} R_{d}\right)
$$

The extrinsic saturation drain voltage can be obtained numerically by equating eq. (15) at $V_{d}=V_{d s a t}$ with eq. (16)

$$
V_{d s a t}=\frac{-\delta_{2}+\sqrt{\delta_{2}^{2}-4 \delta_{1} \delta_{3}}}{2 \delta_{1}}
$$

Where

$$
\begin{aligned}
& \delta_{1}=\frac{Q}{P}\left(A \frac{Q}{P}+\frac{d}{E_{C}}\right)-\frac{\varepsilon \mu Z}{2}\left(2 \frac{Q}{P} R_{d}+1\right) \\
& \delta_{2}=\left(V_{g s}-V_{t h}\right)\left[\frac{Q}{P}\left(R_{d} \varepsilon \mu Z-\frac{d}{E_{C}}\right)+\varepsilon \mu Z\right]-\frac{Q}{P}\left[2\left(V_{g s}-V_{t h}\right) A \frac{Q}{P}-d L-R_{s s} \varepsilon \mu Z V_{g s}+R_{s s} \varepsilon \mu Z V_{t h}\right] \\
& \delta_{3}=\frac{Q}{P}\left(V_{g s}-V_{t h}\right)\left(A \frac{Q}{P} \varepsilon \mu Z-d L-R_{s s} \varepsilon \mu Z V_{g s}+R_{s s} \varepsilon \mu Z V_{t h}\right)
\end{aligned}
$$


International Journal of Advanced Science and Technology

And

$Q=\frac{Z \varepsilon V_{s a t}}{d}, P=1-R_{d} Q$ and $R_{s s}=R_{s}+R_{d}$

2.2.3. Transconductance: Transconductance in the linear region can be obtained by differentiating eq. (15) w.r.t. $V_{g}$

$$
g_{m}=\frac{1}{2 B_{1}}\left[-\varepsilon \mu Z\left(R_{s}+R_{d}\right)+\frac{1}{2 \sqrt{B_{2}^{2}-4 B_{1} B_{2}}}\left\{2 B_{2}\left(-\varepsilon \mu Z\left(R_{s}+R_{d}\right)\right)-4 B_{1} \varepsilon \mu Z V_{d s}\right\}\right]
$$

2.2.4. Drain conductance: The drain conductance can be obtained numerically by differentiating eq. (15) w.r.t. $V_{d}$

$$
g_{d}=\frac{1}{2 B_{1}}\left[\left\{-\frac{d}{E_{c}}-\varepsilon \mu Z\left(2 R_{s}+R_{d}\right)+\frac{1}{2 \sqrt{B_{2}^{2}-4 B_{1} B_{3}}}\left\{2 B_{2}\left\{-\frac{d}{E_{c}}-\varepsilon \mu Z\left(2 R_{s}+R_{d}\right)-4 B_{1}\left(\varepsilon \mu Z\left(V_{g s}-V_{t h}-V_{d s}\right)\right\}\right]\right.\right.\right.
$$

\section{Capacitance-Voltage Characteristics}

Total channel charge is given [17] as:

$$
Q=\int_{0}^{t} I_{d s} d t=\int_{0}^{L} \frac{I_{d s}}{v(x)} d x
$$

Rearranging (2) and using (3), we get

$$
\frac{I d s}{v(x)}=\frac{Z \varepsilon(m)}{d}\left(V_{g s}-V_{t h}-V_{c}(x)\right)
$$

Integrating eq. (21)

$$
\int_{0}^{L} \frac{I_{d s}}{v(x)} d x=\frac{Z \varepsilon(m)}{d} \int_{0}^{L}\left(V_{g s}-V_{t h}-V_{c}(x)\right) d x
$$

Using [18]

$$
d x=\frac{\operatorname{Zqn}_{s}(x) \mu-\frac{I_{d s}}{E_{1}}}{I_{d s}} d V_{c}(x)
$$

Substituting eq. (23) into eq. (22), total charge is given as:

$$
Q=\frac{z \varepsilon(m)}{d} \int_{0}^{L}\left(V_{g s}-V_{t h}-V_{c}(x)\right)\left\{\frac{Z q n_{s}(x) \mu_{0}-\frac{I_{d s}}{E_{3}}}{I_{d s}}\right\} d V_{c}(x)
$$


On solving eq. (24),

$$
Q=\frac{-z^{2} \varepsilon^{2}(m) \mu_{0}}{3 d^{2} I_{d s}}\left[\left(V_{g s}-V_{t h}-V_{d s}\right)^{3}-\left(V_{g s}-V_{t h}\right)^{3}\right]-\frac{Z \varepsilon(m)}{d E_{3}} V_{d s}
$$

\subsection{Gate-Drain Capacitance}

It is also called as feedback capacitance. It is defined as the rate of change of charge on the gate electrode with respect to the drain bias when the source and the gate potentials are kept constant.

$$
C_{d s}=\frac{\partial Q}{\partial V_{g s}}=\frac{R}{I_{d s}}\left(V_{g s}-V_{t h}-V_{d s}\right)^{3}-\frac{Z \varepsilon(m)}{d E_{3}}
$$

\subsection{Gate-Source Capacitance}

The gate-source capacitance $\left(C_{g s}\right)$ is defined as the change in total charge with the change in gate voltage. It is mainly due to the $2 \mathrm{DEG}$ in the normal operating region, and is given as

$$
C_{g s}=\frac{\partial Q}{\partial V_{g s}}=\frac{R}{I_{d s}}\left[\left(V_{g s}-V_{t h}\right)^{2}-\left(V_{g s}-V_{t h}-V_{d s}\right)^{2}\right]
$$

\section{Cut-off Frequency}

It is one of the primary figure of merit for microwave performance, which can be obtained as;

$$
f_{c}=\frac{g_{m}}{2 \pi C_{g}}
$$

Where

$C_{g}=\frac{\varepsilon(m) L Z}{d}=$ effective gate capacitance for linear region.

$\mathrm{LZ}$ is the effective area under the gate.

Finally, the parameters of the proposed model are obtained analytically in the above sections.

\section{Results and Discussion}

The characteristics of AlGaN/GaN MODFET have been modeled and the results obtained are compared with the previous simulated results to prove the validity of the model. The intrinsic and extrinsic characteristics are also plotted to predict the effect of parasitic resistances. 


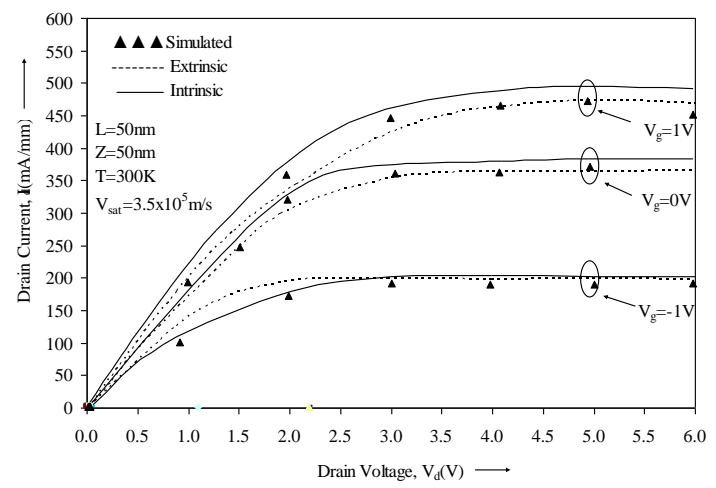

\section{Figure 2. Dependence of Drain Current on Drain Voltage for different values of gate voltage}

The variation of drain current with drain voltage for intrinsic and extrinsic cases at different gate voltages is depicted in Figure 2. A small variation in extrinsic and intrinsic characteristics is observed for smaller gate voltages. The difference between intrinsic and extrinsic characteristics shows that the analysis without parasitic source and drain resistances, i.e., intrinsic case results in low saturation voltage and higher drain currents. Taking into account these parasitic resistances, i.e., extrinsic case, close agreement with the simulated results are obtained. This is because increase in channel resistance as gate voltage decrease. Due to increase in channel resistance, the effect of parasitic resistance becomes negligible. A high saturation current of $502.6 \mathrm{~mA} / \mathrm{mm}$ at a gate bias of $1 \mathrm{~V}$ is achieved. The calculated results are in good agreement with the simulated results [19].

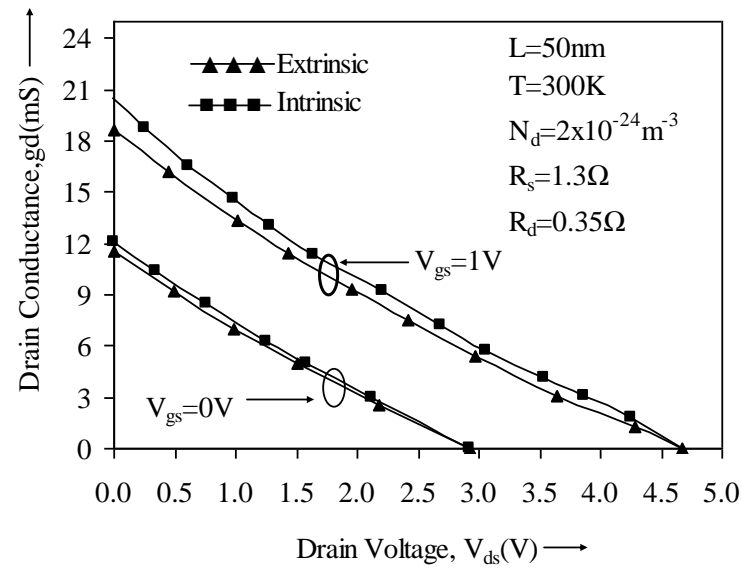

Figure 3. Variation of drain conductance with drain voltage for various gate bias

Figure 3 shows the dependence of output conductance on drain voltage for different gate biases. As depicted in the figure, with the increases in drain voltage, the drain/output conductance decreases sharply and finally becomes zero. The $g_{d}$ decreases with increases in $V_{g}$, the channel resistance decreases and the $g_{d}$ increases with increase in gate voltage. 


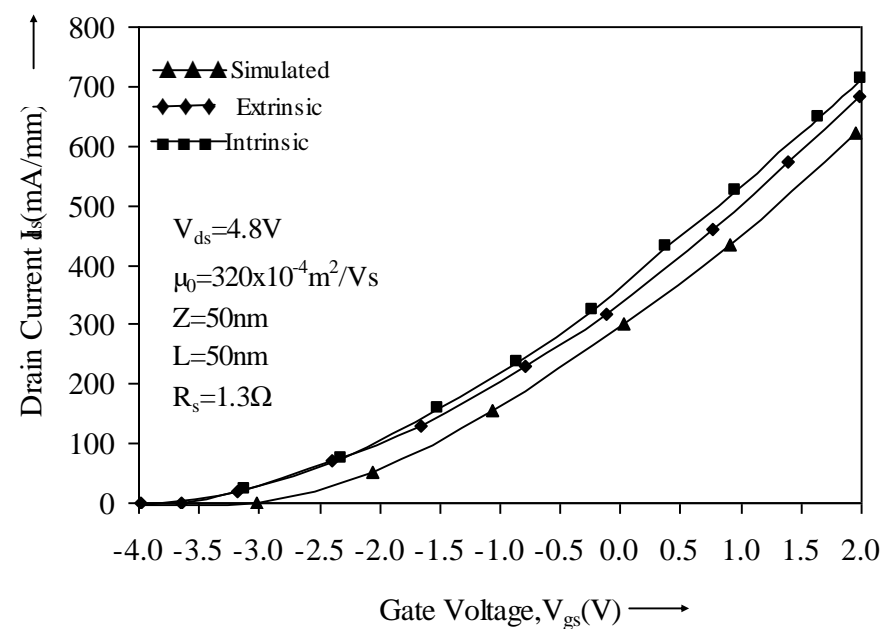

Figure 4. Dependence of drain current on gate voltages

The variation of drain current with gate voltage is depicted in Figure 4. Figure shows that with increase in gate voltage, the drain current also increases. The extrinsic characteristics are found to be closer to the simulated results. The discrepancy observed at higher gate voltages. The analytical results are in close proximity with the previous published results [19].

The variation of transconductance with gate voltage is shown in Figure 5. The transconductance increases with gate voltage in the linear region. The transconductance decreases with the increase in negative gate bias as the depletion layer width increases and channel width decreases. The transconductance of $33.2 \mathrm{mS}$ and $32.3 \mathrm{mS}$ respectively are observed for intrinsic and extrinsic cases.

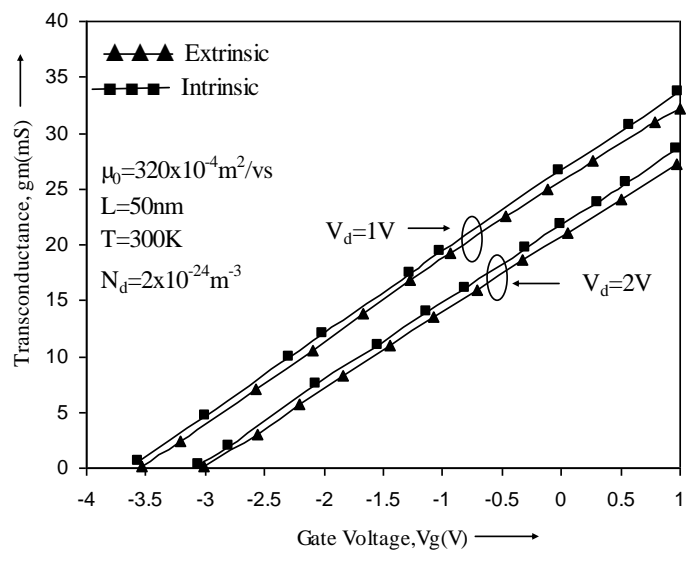

Figure 5. Dependence of Transconductance on Gate Voltage 


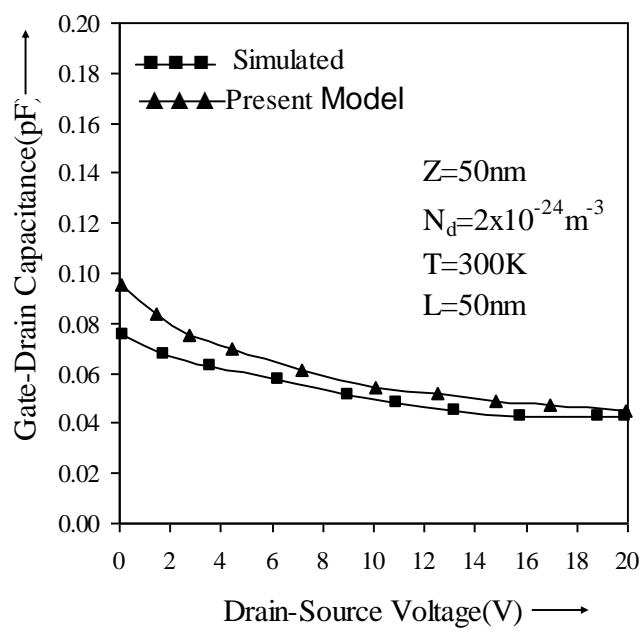

\section{Figure 6. Dependence of gate-drain capacitance on drain source voltage}

The variation of gate-drain capacitance with drain bias is depicted in Figure 6. It can be observed from the figure that the capacitance decreases gradually with increase in drain voltage. The calculated results are in good agreement with the previously simulated results [20].

The dependence of gate-source capacitance on gate voltage is shown in Figure 7. It is evident from the Figure 7 that $C_{g s}$ decreases with increase in negative gate bias because of the increase in depletion layer width. The analytical results are in good agreement with the previously published results [20].

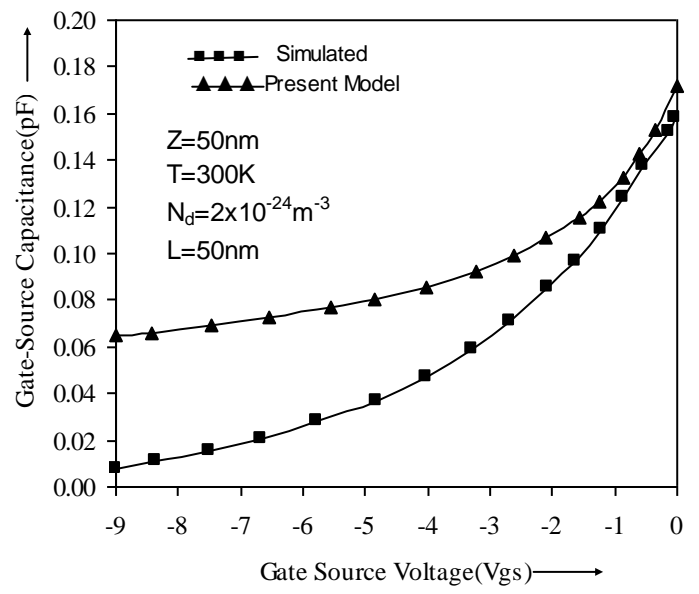

Figure 7. Variation of gate-source capacitance with gate-source voltage 


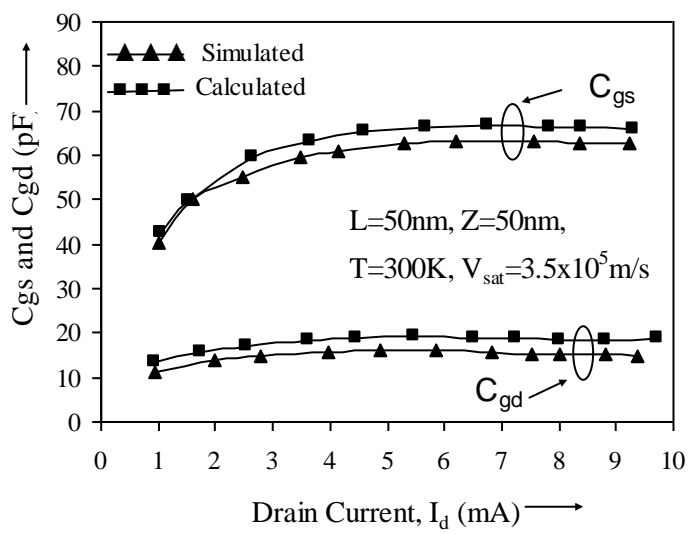

\section{Figure 8. Dependence of gate to source capacitance and gate to drain capacitance on drain current}

The variation of gate-source capacitance and gate-drain capacitance with drain current is shown in Figure 8. It can be seen from the figure that the gate-source capacitance increases with increase in the saturation current because of the increase in gate-source voltage. Also gate-source capacitance is almost constant in the saturation region. The calculated results are in close agreement with the previously published results [21].

The dependence of cutoff frequency with gate voltage for both extrinsic and intrinsic cases is plotted in Figure 9. The cutoff frequency increases with gate voltage and then saturates. The highest cutoff frequencies for both intrinsic and extrinsic cases are observed at $22.5 \mathrm{GHz}$ and $21.3 \mathrm{GHz}$, respectively.

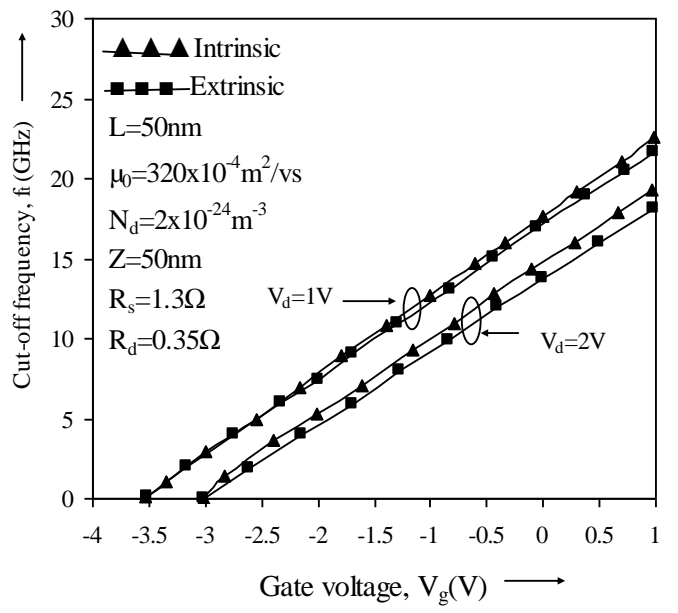

Figure 9. Variation of cut-off frequency with gate voltage

In nutshell, it is observed from the above figures that the results are in close agreement with the previously published results and hence prove the validity of the model.

\section{Conclusion}


A compact and accurate analytical model is developed for the I-V characteristics and small-signal parameters of an AlGaN/GaN MODFET. The model presented in this paper includes the effects of parasitic resistances $R_{s}$ and $R_{d}$ into account. The model showed a high saturation current of $502.6 \mathrm{~mA} / \mathrm{mm}$ at a gate bias of $1 \mathrm{~V}$. A high cut-off frequency of 22.5 $\mathrm{GHz}$ is obtained at a drain bias of $1 \mathrm{~V}$ for a $50 \mathrm{~nm}$ gate length. The expression obtained are fully analytical and do not have any fitting parameters. The need for incorporating the effect of parasitic resistance at higher gate, lower drain voltages and submicron gate length of the device is emphasized. The obtained results are in close agreement with the previously published results proving the validity of the model. The results indicate that the AlGaN/GaN MODFET is suitable for microwave and millimeter wave circuit applications.

\section{References}

[1] A. Ahlawat, M. Pandey and S. Pandey, "Microwave analysis of 70nm InGaAs pHEMT on InP substrate for nanoscale digital IC applications", MOTL, vol. 49, no. 10, (2007).

[2] T. R. Lenka and A. K. Panda, "Characteristics study of modulation doped GaAs/InGaAs/AlGaAs based Pseudomorphic HEMT", International journal of recent trends in Engineering, vol. 1, no. 3, (2009).

[3] A. Agrawal, A. Goswami, S. Sen and R. S. Gupta, "Transconductance extraction for pseudomorphic MODFET (AlGaAs/InGaAs) for microwave and millimeter wave applications”, MOTL, vol. 22, (1999).

[4] M. K. Chattopadhyay and S. Tokekar, "Analytical model for the transconductance of microwave AlGaN/GaN HEMTs including nonlinear macroscopic polarization and parasitic MESFET conduction”, MOTL, vol. 49, no. 2, (2007).

[5] C. Campbell, C. Lee, V. Williams, M. -Y. Kao, H.-Q. Tserng, P. Saunier and T. balisteri, "A wideband power amplifier MMIC utilizing GaN on SiC HEMT technology", IEEE Journal of solid-state circuits, vol. 44, no. 10, (2009).

[6] Z. Hemaizia, N. Sengouga and M. Missous, "Small signal modeling of PHEMTS and analysis of their microwave performance", Courrier du savoir, vol. 10, (2010).

[7] L. Wang, R. -M. Xu and B. Yan, "Accurate small-signal model extraction for pHEMT on GaAs", Int J Infrared Milli Waves, vol. 28, (2007).

[8] M. Bhattacharya, J. Jogi, R. S. Gupta, M. Gupta, "Scattering parameter based modeling and simulation of symmetric tied-gate InAlAs/InGaAs DG-HEMT for millimeter-wave applications", Solid-State Electronics, vol. 63, (2011).

[9] M. Nawaj, “A new charge conserving capacitance model for HEMTs for CAD applications", Int. J. Electronics, vol. 83, no. 6, (1997).

[10] P. Ghosh, S. Haldar, R. S. Gupta and M. Gupta, "An accurate small signal modelling of cylindrical/surrounded gate MOSFET for high frequency applications", JSTS, vol. 12, no. 4, (2012).

[11] Y. Cai, Y. Zhou, K. M. Lau and K. J. Chen, "Contronl of threshold voltage of AlGaN/GaN HEMTs by Fluoride-based plasma treatment from depletion mode to enhancement mode", IEEE Transactions on Electron Devices, vol. 53, no. 9, (2006).

[12] P. K. Chopra, S. Jain and A. Ahlawat, "ANN modeling approach for designing low noise PHEMT amplifier in wireless communication systems", Optical Memory and Neyral Networks(Information Optics), vol. 20, no. 4, (2011).

[13] R. K. Tyagi, A. Ahlawat, M. Pandey and S. Pandey, "Noise analysis of sub-quarter micrometer AlGaN/GaN microwave power HEMT", Journal of Semiconductor Technology and Science, vol. 9, no. 3, 2009), pp. 125135.

[14] D. -H. and J. A. Del Alamo, "30-nm InAs PHEMTs with $\mathrm{f}_{\mathrm{T}}=644 \mathrm{GHz}$ and $\mathrm{f}_{\max }=681 \mathrm{GHz}$ ", IEEE Electron Device Letters, vol. 31, no. 8, (2010), pp. 806-808.

[15] F. Yulong, D. Shaobo, L. Bo, Y. Jiayun, C. Shujun and F. Zhihong, "Extrinsic and Intrinsic causes of the electrical degradation of AlGaN/GaN high electron mobility transistors", Journal of Semiconductors, vol. 33, no. 5, (2012), pp. 054005-1-4.

[16] N. Dasgupta and A. Dasgupta, "An analytic expression for sheet carrier concentration vs. gate voltage for HEMT modeling”, Solid-State electronics, vol. 36, (1993), pp. 201-203.

[17] P. Gangwani, et al., "Polarization dependent analysis of AlGaN/GaN HEMT for high power applications", Elsevier, ScienceDirect, Solid-State Electronics, vol. 51 (2007). 
[18] S. Sen, et al., "Two-Dimentional C-V Model of AlGaAs/GaAs Modulation Doped Field EffectTransistor (MODFET) for High Frequency Applications", IEEE Transactions on Electron Devices, vol. 46, no. 9, (1999).

[19] J. C. Sippel, S. S. Islam and S. S. Mukherjee, "A physics based model of DC and microwave characteristics of GaN/AlGaN HEMTs”, International journal of RF and Microwave Computer aided Engineering, vol. 17, (2007).

[20] S. Bose, Adarsh, A. Kumar, Simrata, M. Gupta and R. S.Gupta, "A complete analytical model of GaN MESFET for microwave frequency applications", Microelectronics Journal, vol. 32, (2001).

[21] A. Goswami, M. Gupta and R. S. Gupta, "Analysis of scattering parameters and thermal noise of a MOSFET for its microwave frequency applications", MOTL, vol. 31, no. 2, (2001).

\section{Authors}

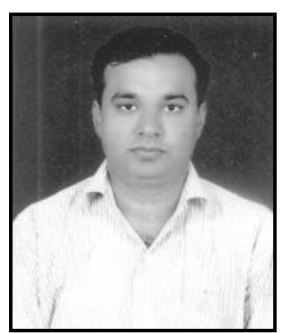

\section{Ramnish}

He received his B.E. (Electronics and Communication) from C.R.S.C.E. (now DCRUST), Murthal, Sonepat, Haryana; M.Tech. from R.E.C., Kurukshetra, Haryana. Presently, he is working as an Assistant Professor in Department of E.C.E., G.J.U.S. \&T., Hisar, Haryana. His present research interests include device modeling, control system and Digital Signal Processing.

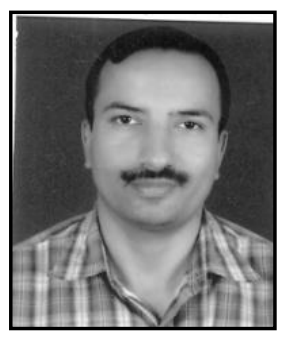

\section{Sandeep k Arya}

He received his B.Tech. (E.C.E.), M.Tech. (E.C.E.), Ph.D from N.I.T., kurukshetra . He has published more than 100 research papers in international/National journals/ Conferences. Presently, he is working as a Professor and Head of Department (E.C.E.), G.J.U.S. \&T., Hisar since 2004. His research interests include optical communication, Digital Signal Processing and VLSI.

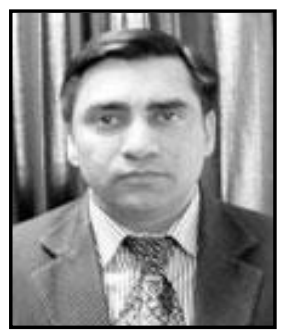

\section{Anil Ahlawat}

He received his M.Sc. degree in Physics (Electronics) from CCS University, Meerut, U.P.; M.Phil.(Instrumentation) from I.I.T., Roorkee and M.Tech.(CSE) from K.U., Kurukshetra, Haryana. He has completed his Ph.D degree from University School of Engineering and Technology, Guru Gobind Singh IndraPrastha University, New Delhi, India. He has published more than 50 research papers in international/National journals/ Conferences. He is presently working as a Professor and Head in Department of C.S.E.in K.I.E.T., Ghaziabad, U.P., India. His research interests include device modeling, simulation of high electron mobility transistors, artificial Neural Networks and Artificial Intelligence. 
International Journal of Advanced Science and Technology Vol.66 (2014) 